\title{
Acute Fasciola hepatica infection attributed to chewing khat
}

Background-Fascioliasis is a parasitic infection, primarily of the liver but after several weeks the biliary tract may also be affected, related to the trematode Fasciola hepatica. The trematode is distributed worldwide in herbivores but is unusual in humans. Several outbreaks have been attributed to consumption of contaminated watercress and water. The optimal treatment of fascioliasis, particularly the acute form, is still unknown. We describe a patient with an acute Fasciola hepatica infection following a rare transmission route, who was successfully treated with a simple and safe drug regimen.

Case report - A 36 year old previously healthy Somalian man was admitted to our department with a one week history of right hypochondrial pain. The pain resolved spontaneously within three days. He had come to live in the Netherlands in 1986, and since then had only visited Saudi Arabia for pilgrimage one year before admission. Physical examination revealed no abnormalities. Full blood count showed leucocytosis $\left(16.4 \times 10^{9} / 1\right)$ with abundant eosinophils $(51 \%)$. Liver function tests showed marginally increased alkaline phosphatase (141 U/l), and normal transaminases and alpha fetoprotein. Abdominal ultrasonography and computed tomography scan revealed a large irregular mass in the left liver lobe with multiple hilar lymph nodes suggestive of malignancy (fig 1). Histology of liver biopsy specimens obtained via diagnostic laparoscopy revealed a non-caseating granulomatous eosinophilic infiltrate without evidence of malignancy. Two different serological tests for Fasciola hepatica showed serum titres greater than 1:256. Several examinations of the stool revealed no ova. The patient reported that he had never eaten liver or watercress or other wild waterplants. However, he chewed khat leaves (Catha edulis) containing psychoactive compounds on weekends. The khat was freshly imported from Kenya. He was treated for acute fascioliasis with a single dose of triclabendazole $700 \mathrm{mg}$ (12 $\mathrm{mg} / \mathrm{kg}$ ); no complications occurred. Serological follow up showed complete disappearance of serum antibodies one year after therapy. Control imaging series revealed resolution of the intrahepatic mass and lymph nodes (fig 2).

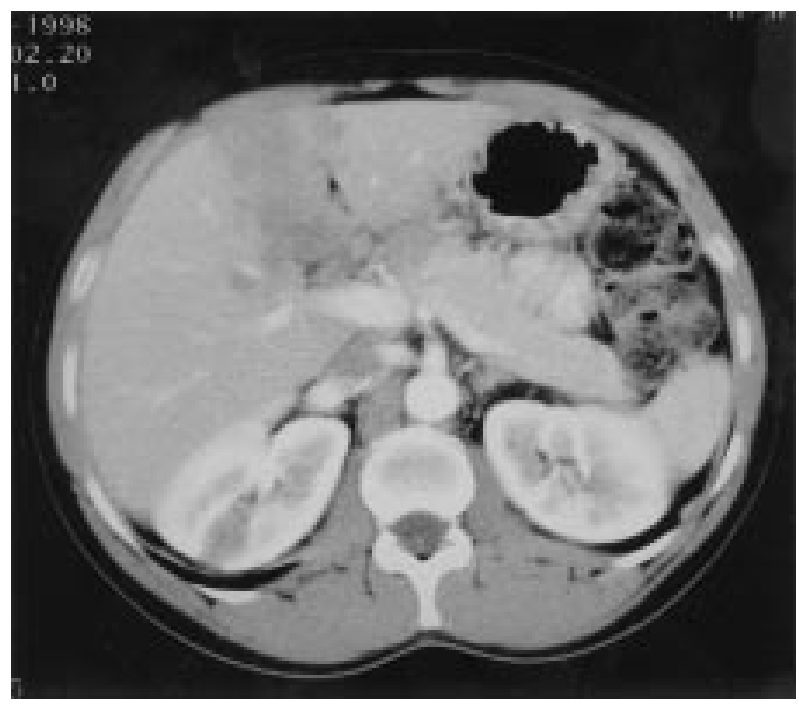

Figure 1 Computed tomography scan with oral and intravenous contrast showing a large irregular mass in the left liver lobe with several hilar lymph nodes. A small cyst is seen in the right kidney also.
Discussion - The life cycle of Fasciola hepatica includes a sexual phase in freshwater snail (intermediate host) and a sexual phase in mammals

(definitive host); humans are an accidental host. In mammals, following digestion, Fasciola hepatica subsequently invade the gut wall, peritoneal cavity, hepatic capsule, and hepatic parenchyma, and finally reside in the biliary tract where locally produced eggs are excreted with faeces. The eggs hatch in freshwater into ciliated miracidia that invade snails. The cercariae emerging from snails encyst on waterplants and, under moist conditions, can survive for long periods of time. Human infection usually occurs after consumption of contaminated watercress or water, and theoretically can take place after consuming contaminated liver. In fact, only one patient described in the literature was thought to be infected otherwise. ${ }^{1}$ In a Yemenite woman residing in the UK, five years after the onset of abdominal pain, chronic infection with Fasciola hepatica was established and attributed to previous khat consumption. In our case, in the absence of other transmission routes, chewing contaminated imported khat was most likely the route of infection. It appeared that the khat shrubs were cultivated in areas housing sheep, and were irrigated with local water. Furthermore, freshly plucked khat leaves were kept damp and wrapped in banana leaves during transport. This may prolong the survival time of encysted metacercariae.

Triclabendazole, a chloro-phenoxy benzimidazole compound, given as a single dose, has been reported to be highly effective against both the acute migratory and chronic form of fascioliasis in cattle and sheep. It has also been proposed as the drug of choice in chronic human fascioliasis. ${ }^{2}$ Experience with triclabendazole in acute human Fasciola hepatica infection however is limited. Two case reports described successful treatment of acute infection. ${ }^{34}$ In our patient, a favourable outcome was observed.

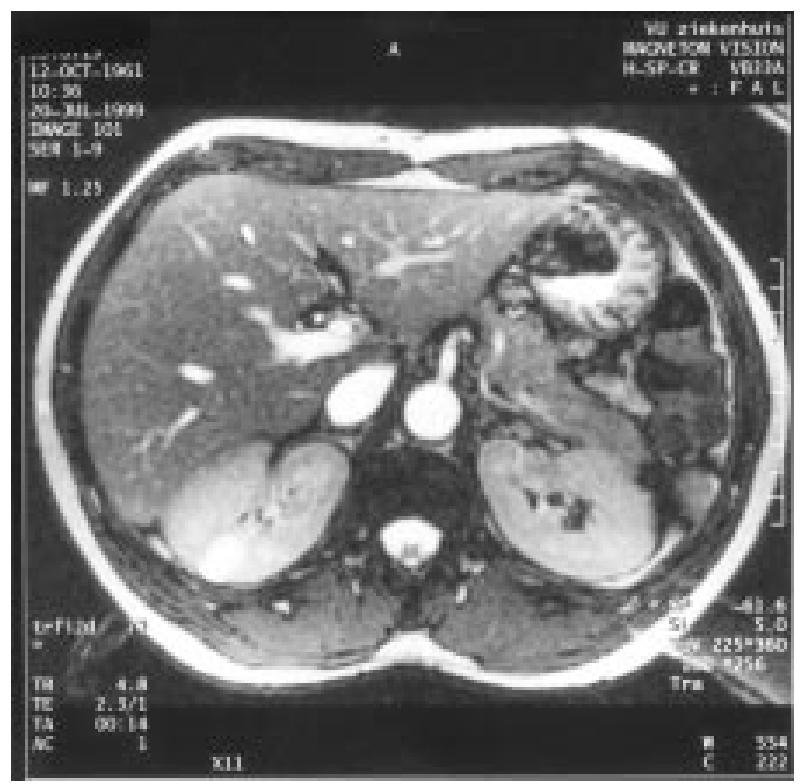

Figure 2 Magnetic resonance image obtained nine months after treatment showing no abnormalities in the liver. The same cyst in the right kidney is observed. 
Conclusion-Fascioliasis can be due to chewing contaminated khat. Treatment with a single dose of triclabendazole is safe and effective.

Department of Gastroenterology, Academic Hospital Vrije Universiteit, Amsterdam, Netherlands

A CATS P SCHOLTEN $S$ G M MEUWISSEN E J KUIPERS
Correspondence to: Dr A Cats, Department of Gastroenterology, Academic Hospital Vrije Universiteit, PO Box 7057, 1007 MB Amsterdam, Netherlands. Email: a.cats@azvu.nl

1 Doherty JF, Price N, Moody AH, et al. Fasciola due to imported khat. Lan-

2 Apt W, Aguilera X, Vega F, et al. Treatment of human chronic fascioliasis with triclabendazole: drug efficacy and serologic response. Am $\mathcal{F}$ Trop Med Hyg 1995;52:532-5.

3 Loutan L, Bouvier M, Rojanawisut B, et al. Single treatment of invasive fascioliasis with triclabendazole. Lancet 1989;2:383.

4 Picot S, Querrec M, Ghez JL, et al. A new report of triclabendazole efficacy during invading phase fasciolasis. Eur f Clin Microbiol 1992;11:269-70. 